\title{
Multiple solutions for a problem with discontinuous nonlinearity
}

\author{
Claudianor O. Alves ${ }^{1} \cdot$ Jefferson A. dos Santos ${ }^{1}$. \\ Rodrigo C. M. Nemer ${ }^{1}$
}

Received: 10 February 2017 / Accepted: 16 October 2017 / Published online: 27 October 2017

(C) Fondazione Annali di Matematica Pura ed Applicata and Springer-Verlag GmbH Germany 2017

\begin{abstract}
In this work, we use the Lusternik-Schnirelmann category to estimate the number of nontrivial solutions for a problem with discontinuous nonlinearity and subcritical growth.
\end{abstract}

Keywords Variational methods · Discontinuous nonlinearity · Lusternik-Schnirelmann category

Mathematics Subject Classification 35A15 14 E20 $\cdot 35 \mathrm{H} 30 \cdot 35 \mathrm{Q} 55$

\section{Introduction}

The present work studies the existence of multiple solutions for the following class of discontinuous problems

$$
\left\{\begin{array}{l}
-\Delta u=f_{p, \delta}(u(x)), \quad \text { a.e in } \quad \Omega, \\
u \in W^{2, \frac{p}{p-1}}(\Omega) \cap H_{0}^{1}(\Omega),
\end{array}\right.
$$

where $\Omega \subset \mathbb{R}^{N}(N \geq 3)$ is a smooth bounded domain, $f_{p, \delta}: \mathbb{R} \rightarrow \mathbb{R}$ is the odd function given by

$$
f_{p, \delta}(t)= \begin{cases}t|t|^{p-2}, & t \in[0, a], \\ (1+\delta) t|t|^{p-2}, & t>a .\end{cases}
$$

with $a, \delta>0$ and $p \in\left(2,2^{*}\right)$.

C.O. Alves was partially supported by CNPq/Brazil 301807/2013-2.

Jefferson A. dos Santos

jeffer.abrantes@gmail.com

1 Unidade Acadêmica de Matemática, Universidade Federal de Campina Grande, Campina Grande, PB 58429-900, Brazil 
In [14], Benci and Cerami have considered the existence of multiple positive solutions for the case $\delta=0$, that is, for the problem

$$
\left\{\begin{array}{l}
-\Delta u=|u|^{p-2} u, \quad x \in \Omega, \\
u \in H_{0}^{1}(\Omega) .
\end{array}\right.
$$

By using variational methods combined with the Lusternik-Schnirelmann category, Benci and Cerami proved that if $p$ is close to $2^{*}=\frac{2 N}{N-2}$, then problem $\left(P_{p, 0}\right)$ has at least $\operatorname{cat}(\Omega)$ of positive solutions. Here, we recall that if $X$ is a topological space and $A \subset X$ a closed subspace, we denote by $\operatorname{cat}_{X}(A)$ the Lusternik-Schnirelmann category of $A$ in $X$. The Lusternik-Schnirelmann category, $\operatorname{cat}_{X}(A)$, is the least number of closed and contractible sets in $X$ which cover $A$. If $X=A$, we use the short notation $\operatorname{cat}(X)$. Later, Benci and Cerami [15] generalized their previous result by working with a more general nonlinearity and Morse theory.

The reader can find in the literature a lot of papers where the existence and multiplicity of solutions for related problems to $\left(P_{p, 0}\right)$ are directly associated with the topological richness of $\Omega$, see Alves and Ding [9], Bahri and Coron [13], Rey [29], Struwe [31] and their references.

For the case $\delta>0$, the function $f_{p, \delta}$ is discontinuous and the study of existence of solution for $\left(P_{p, \delta}\right)$ is totally different of the case $\delta=0$, because we cannot use directly the results for $C^{1}$-functionals, then the existence and multiplicity of solution for $\left(P_{p, \delta}\right)$ associated with the topological richness of $\Omega$ is an open and interesting problem. Motivated by this fact, in the present paper we prove a result of multiplicity of solutions in the same spirit of [14]; more precisely, we prove that if $\delta$ is small enough and $p$ is close to $2^{*}$, the problem $\left(P_{p, \delta}\right)$ has at least $\operatorname{cat}(\Omega)$ of positive solutions, see Theorem 1.1 .

The interest in the study of nonlinear partial differential equations with discontinuous nonlinearities has increased because many free boundary problems arising in mathematical physics may be stated in this form. Among these problems, we have the obstacle problem, the seepage surface problem, and the Elenbaas equation; see, for example, [18-20].

A rich literature is available by now on problems with discontinuous nonlinearities, and we refer the reader to Ambrosetti and Turner [2], Ambrosetti et al. [5], Alves et al. [6], Alves and Bertone [7], Alves et al. [8], Badiale and Tarantelo [12], Carl et al. [16], Clarke [17], Chang [18], Carl and Dietrich [21], Carl and Heikkila [22,23], Cerami [24], Hu et al. [25], Montreanu and Vargas [27], Radulescu [28] and their references. Several techniques have been developed or applied in their study, such as variational methods for nondifferentiable functionals, lower and upper solutions, global branching, fixed point theorem, and the theory of multivalued mappings.

Our main result is the following:

Theorem 1.1 There are $\delta^{*}>0$ and $p^{*} \in\left(2,2^{*}\right)$ such that for each $\delta \in\left(0, \delta^{*}\right)$ and $p \in\left(p^{*}, 2^{*}\right),\left(P_{p, \delta}\right)$ has at least $\operatorname{cat}(\Omega)$ nontrivial solutions.

In the proof of the above result, we will adapt for our case an approach explored by Ambrosetti and Badiale [4]. The main idea consists in setting a suitable single function and then considering a dual functional, which is $C^{1}$ and their critical points produce solutions for $\left(P_{p, \delta}\right)$. For more details, see Sect. 2.

Notations In this paper, we use the following notations:

- For $q \in\left(2,2^{*}\right)$, we define $q^{\prime}$ as the conjugate exponent of $q$, that is, $q^{\prime}=\frac{q}{q-1}$.

- We denote by $2^{+}$the conjugate exponent of $2^{*}=\frac{2 N}{N-2}$, that is, $2^{+}=\frac{2 N}{N+2}$.

- The usual norm of the Lebesgue spaces $L^{t}(\Omega)$ for $t \in[1, \infty]$ will be denoted by $|\cdot|_{t}$ and the norm of the Sobolev space $H_{0}^{1}(\Omega)$, by $\|$.$\| ;$ 
- $C$ denotes (possibly different) any positive constant.

- If $A \subset \mathbb{R}^{N}$ is a measurable set, we denote by meas $(A)$ its Lebesgue measure.

- If $X$ and $Y$ are topological spaces, we say that $X$ and $Y$ are homotopically equivalent if there exist continuous functions $h: X \rightarrow Y$ and $q: Y \rightarrow X$ such that $q \circ h=i d_{X}$ and $h \circ q=i d_{Y}$.

\section{An auxiliary problem}

In the sequel, we consider the energy functional $I_{p, \delta}: H_{0}^{1}(\Omega) \rightarrow \mathbb{R}$ associated with $\left(P_{p, \delta}\right)$ given by

$$
I_{p, \delta}(u)=\frac{1}{2} \int_{\Omega}|\nabla u|^{2} \mathrm{~d} x-\int_{\Omega} F_{p, \delta}(u) \mathrm{d} x,
$$

where

$$
F_{p, \delta}(t)=\int_{0}^{t} f_{p, \delta}(r) \mathrm{d} r .
$$

Notice that $I_{p, \delta}$ is not a differentiable functional, because $F_{p, \delta}$ is only a continuous function. This fact does not allow to use the traditional methods to get multiplicity of solutions by using Lusternik-Schnirelmann category. To avoid this difficulty, we will adapt for our problem an approach explored in Ambrosetti and Badiale [4].

In what follows, we denote by $g_{p^{\prime}, \delta}: \mathbb{R} \rightarrow \mathbb{R}$ the odd function given by

$$
g_{p^{\prime}, \delta}(s)= \begin{cases}s|s|^{p^{\prime}-2}, & s \in\left[0, a^{p-1}\right], \\ a, & s \in\left[a^{p-1},(1+\delta) a^{p-1}\right], \\ (1+\delta)^{-\frac{1}{p-1}} s|s|^{p^{\prime}-2}, & s \in\left[(1+\delta) a^{p-1},+\infty\right) .\end{cases}
$$

The functions $f_{p, \delta}$ and $g_{p^{\prime}, \delta}$ are related in the following way:

(a)

$$
f_{p, \delta}\left(g_{p^{\prime}, \delta}(s)\right)= \begin{cases}s, & s \notin\left[a^{p-1},(1+\delta) a^{p-1}\right], \\ a^{p-1}, & s \in\left[a^{p-1},(1+\delta) a^{p-1}\right] ;\end{cases}
$$

(b) $g_{p^{\prime}, \delta}\left(f_{p, \delta}(t)\right)=t, \forall t \in \mathbb{R}$.

In the sequel, $G_{p^{\prime}, \delta}$ denotes the primitive of $g_{p^{\prime}, \delta}$, that is,

$$
G_{p^{\prime}, \delta}(s):=\int_{0}^{s} g_{p^{\prime}, \delta}(r) \mathrm{d} r .
$$

From definition of $g_{p^{\prime}, \delta}, G_{p^{\prime}, \delta}$ is an even function with

$$
G_{p^{\prime}, \delta}(s)= \begin{cases}\frac{1}{p^{\prime}} s^{p^{\prime}}, & s \in\left[0, a^{p-1}\right], \\ a s-\frac{a^{p}}{p}, & s \in\left[a^{p-1},(1+\delta) a^{p-1}\right], \\ \frac{\gamma_{\delta}}{p^{\prime}} s^{p^{\prime}}+\delta \frac{a^{p}}{p}, & s \in\left[(1+\delta) a^{p-1},+\infty\right),\end{cases}
$$

for $\gamma_{\delta}=(1+\delta)^{-\frac{1}{p-1}}$. Thus,

$$
\gamma_{\delta}|s|^{\frac{1}{p-1}} \leq\left|g_{p^{\prime}, \delta}(s)\right| \leq|s|^{\frac{1}{p-1}}, \quad \forall s \in \mathbb{R}
$$


and

$$
\frac{\gamma_{\delta}}{p^{\prime}}|s|^{p^{\prime}} \leq G_{p^{\prime}, \delta}(s) \leq \frac{1}{p^{\prime}}|s|^{p^{\prime}}, \quad \forall s \in \mathbb{R} .
$$

To simplify the notation, we denote by $g_{p^{\prime}}$ and $G_{p^{\prime}}$ the functions $g_{p^{\prime}, 0}$ and $G_{p^{\prime}, 0}$, respectively.

The next step is to define the dual functional associated with $I_{p, \delta}$. By [26, Theorem 11.3], we know that for each $u \in L^{p^{\prime}}(\Omega)$ there is an unique solution $w \in W_{0}^{1, p^{\prime}}(\Omega) \cap W^{2, p^{\prime}}(\Omega)$ for the problem

$$
\left\{\begin{array}{l}
-\Delta w=u, \quad x \in \Omega, \\
w=0, \quad x \in \partial \Omega .
\end{array}\right.
$$

Moreover, there is a positive constant $C$ independent of $w$ such that

$$
\|w\|_{W^{2, p^{\prime}}(\Omega)} \leq C|u|_{p^{\prime}} .
$$

The above information permits to define a linear operator $K_{p^{\prime}, \Omega}: L^{p^{\prime}}(\Omega) \rightarrow W^{2, p^{\prime}}(\Omega)$, such that for $u \in L^{p^{\prime}}(\Omega), K_{p^{\prime}, \Omega}(u)$ is the unique solution of (2.4). Hence,

$$
\left\|K_{p^{\prime}, \Omega}(u)\right\|_{W^{2, p^{\prime}}(\Omega)} \leq C|u|_{p^{\prime}}, \quad \forall u \in L^{p^{\prime}}(\Omega),
$$

from where it follows that $K_{p^{\prime}, \Omega}$ is continuous. On the other hand, since the embeddings below

$$
W^{2, p^{\prime}}(\Omega) \hookrightarrow L^{s}(\Omega), \quad \forall s \in\left[1,\left(p^{\prime}\right)^{*}\right),
$$

are compact for

$$
\left(p^{\prime}\right)^{*}= \begin{cases}\frac{N p^{\prime}}{N-2 p^{\prime}}, & N>2 p^{\prime}, \\ +\infty, & 1 \leq N \leq 2 p^{\prime},\end{cases}
$$

we can ensure that $K_{p^{\prime}, \Omega}: L^{p^{\prime}}(\Omega) \rightarrow L^{p}(\Omega)$ is a linear compact operator, because $p \in\left(2,2^{*}\right)$ if, and only $p \in\left(2,\left(p^{\prime}\right)^{*}\right)$. Moreover, it is easy to check that

$$
\int_{\Omega} K_{p^{\prime}, \Omega}(u) v \mathrm{~d} x=\int_{\Omega} K_{p^{\prime}, \Omega}(v) u \mathrm{~d} x, \quad \forall u, v \in L^{p^{\prime}}(\Omega) .
$$

Using the above notations, we set the functional $J_{p^{\prime}, \delta}: L^{p^{\prime}}(\Omega) \rightarrow \mathbb{R}$ given by

$$
J_{p^{\prime}, \delta}(u)=\int_{\Omega} G_{p^{\prime}, \delta}(u) \mathrm{d} x-\frac{1}{2} \int_{\Omega} K_{p^{\prime}, \Omega}(u) u \mathrm{~d} x .
$$

The functional $J_{p^{\prime}, \delta}$ is called the Dual functional associated with $I_{p, \delta}$. Observe that, differently of $I_{p, \delta}, J_{p^{\prime}, \delta} \in C^{1}\left(L^{p^{\prime}}(\Omega), \mathbb{R}\right)$ and

$$
J_{p^{\prime}, \delta}^{\prime}(u) v=\int_{\Omega}\left(g_{p^{\prime}, \delta}(u) \mathrm{d} x-K_{p^{\prime}, \Omega}(u)\right) v \mathrm{~d} x, \forall u, v \in L^{p^{\prime}}(\Omega) .
$$

Thus, $u \in L^{p^{\prime}}(\Omega)$ is a critical point of $J_{p^{\prime}, \delta}$ if, and only if,

$$
g_{p^{\prime}, \delta}(u)=K_{p^{\prime}, \Omega}(u) \text { a.e. in } \Omega .
$$

The above equality permits to prove the following proposition:

Proposition 2.1 If $u$ is a critical point of $J_{p^{\prime}, \delta}$, then $v:=g_{p^{\prime}, \delta}(u)$ is a solution of the problem $\left(P_{p, \delta}\right)$. 
Proof If $u$ is a critical point for $J_{p^{\prime}, \delta}$, then

$$
v(x)=K_{p^{\prime}, \Omega}(u(x)) \text { a.e. in } \Omega,
$$

from where it follows that

$$
-\Delta v(x)=u(x) \quad \text { a.e. in } \Omega \text {. }
$$

Thereby, if $|v(x)| \neq a$,

$$
-\Delta v(x)=u(x)=f_{p, \delta}\left(g_{p^{\prime}, \delta}(u(x))\right)=f_{p, \delta}(v(x)) .
$$

If $|v(x)|=a$, we have that

$$
-\Delta v(x)=0, \quad \text { a.e. in } \quad \mathcal{A}=\{x \in \Omega:|v(x)|=a\} .
$$

On the other hand, $v(x)=g_{p^{\prime}, \delta}(u(x))$ and if $|v(x)|=a$ then necessarily $u(x) \neq 0$, by definition of $g_{p^{\prime}, \delta}$. Then,

$$
-\Delta v(x)=u(x) \neq 0 \text { a.e. in } \mathcal{A} \text {. }
$$

From (2.6)-(2.7), it follows that $\mathcal{A}$ has measure zero. Therefore,

$$
-\Delta v(x)=f_{p, \delta}(v(x)), \quad \text { a.e in } \Omega \text { and } v \in H_{0}^{1}(\Omega) .
$$

Now, the elliptic regularity gives $v \in W^{2, \frac{p}{p-1}}(\Omega)$, showing that $v$ is a solution of $\left(P_{p, \delta}\right)$.

Motivated by the last proposition, we will look for critical points of $J_{p^{\prime}, \delta}$. The result below establishes that $J_{p^{\prime}, \delta}$ satisfies the mountain pass geometry.

Proposition 2.2 The functional $J_{p^{\prime}, \delta}$ has the mountain pass geometry, that is,

(i) $J_{p^{\prime}, \delta}(0)=0$ and there is $\rho>0$ such that

$$
\inf _{|u|_{p^{\prime}}=\rho} J_{p^{\prime}, \delta}(u)>0 \text { and } J_{p^{\prime}, \delta}(u) \geq 0, \forall u \in L^{p^{\prime}}(\Omega) \text { with }|u|_{p^{\prime}} \leq \rho .
$$

(ii) There is $\psi \in L^{p^{\prime}}(\Omega)$ such that

$$
|\psi|_{p^{\prime}}>\rho \text { and } J_{p^{\prime}, \delta}(\psi)<0
$$

Proof We begin by showing $(i)$. The equality $J_{p^{\prime}, \delta}(0)=0$ is immediate. From (2.3),

$$
\int_{\Omega} G_{p^{\prime}, \delta}(u) \mathrm{d} x \geq \frac{\gamma_{\delta}}{p^{\prime}}|u|_{p^{\prime}}^{p^{\prime}}, \quad \forall u \in L^{p^{\prime}}(\Omega),
$$

and by Hölder's inequality and continuity of $K_{p^{\prime}, \Omega}$, there is $C>0$ such that

$$
\int_{\Omega} K_{p^{\prime}, \Omega}(u) u \mathrm{~d} x \leq C|u|_{p^{\prime}}^{2}, \quad \forall u \in L^{p^{\prime}}(\Omega) .
$$

Thus, (2.8) and (2.9) combine to give

$$
\begin{aligned}
J_{p^{\prime}, \delta}(u) & \geq \frac{\gamma_{\delta}}{p^{\prime}}|u|_{p^{\prime}}^{p^{\prime}}-\frac{C}{2}|u|_{p^{\prime}}^{2} \\
& =|u|_{p^{\prime}}^{p^{\prime}}\left(\frac{\gamma_{\delta}}{p^{\prime}}-\frac{C}{2}|u|_{p^{\prime}}^{2-p^{\prime}}\right) .
\end{aligned}
$$


Since $p^{\prime}<2$, there is $\rho>0$ as in $(i)$. For $(i i)$, notice that for each $\tilde{\psi} \in C_{0}(\Omega) \backslash\{0\}$,

$$
\lim _{t \rightarrow \infty} J_{p^{\prime}, \delta}(t \tilde{\psi})=-\infty .
$$

Therefore, for $t_{0}>0$ large enough, $\psi:=t_{0} \tilde{\psi}$ is as required in (ii).

The next proposition is crucial in our argument, because it proves that $J_{p^{\prime}, \delta}$ verifies the $(P S)$ condition for $\delta$ small enough.

Proposition 2.3 There is $\delta_{0}>0$ such that for all $\delta \in\left[0, \delta_{0}\right]$, the functional $J_{p^{\prime}, \delta}$ satisfies the $(P S)$ condition, that is, if $\left(u_{n}\right) \subset L^{p^{\prime}}(\Omega)$ is such that

$$
\sup _{n \in \mathbb{N}}\left|J_{p^{\prime}, \delta}\left(u_{n}\right)\right|<\infty \text { and } J_{p^{\prime}, \delta}^{\prime}\left(u_{n}\right) \rightarrow 0 \text { as } n \rightarrow \infty,
$$

then there is $u \in L^{p^{\prime}}(\Omega)$ such that, up to a subsequence, $u_{n} \rightarrow u$ in $L^{p^{\prime}}(\Omega)$.

Proof Let $\left(u_{n}\right) \subset L^{p^{\prime}}(\Omega)$ be a sequence with

$$
\sup _{n \in \mathbb{N}}\left|J_{p^{\prime}, \delta}\left(u_{n}\right)\right|<\infty \text { and } J_{p^{\prime}, \delta}^{\prime}\left(u_{n}\right) \rightarrow 0 .
$$

Taking a subsequence if necessary, we can assume that $J_{p^{\prime}, \delta}\left(u_{n}\right) \rightarrow d$ as $n \rightarrow \infty$, and so $\left(u_{n}\right)$ is a bounded sequence in $L^{p^{\prime}}(\Omega)$. Indeed, for $n$ large enough,

$$
d+1+\left|u_{n}\right|_{p^{\prime}} \geq J_{p^{\prime}, \delta}\left(u_{n}\right)-\frac{1}{2} J_{p^{\prime}, \delta}^{\prime}\left(u_{n}\right) u_{n}=\int_{\Omega}\left(G_{p^{\prime}, \delta}\left(u_{n}\right)-\frac{1}{2} g_{p^{\prime}, \delta}\left(u_{n}\right) u_{n}\right) \mathrm{d} x .
$$

As $g_{p^{\prime}, \delta}$ and $G_{p^{\prime}, \delta}$ are odd and even functions, respectively, (2.2) and (2.3) ensure that

$$
G_{p^{\prime}, \delta}(t)-\frac{1}{2} \operatorname{tg}_{p^{\prime}, \delta}(t) \geq\left(\frac{\gamma_{\delta}}{p^{\prime}}-\frac{1}{2}\right)|t|^{p^{\prime}}, \quad \forall t \in \mathbb{R} .
$$

Once $p^{\prime}<2$ and $\gamma_{\delta}=(1+\delta)^{-\frac{1}{p-1}}$, there is $\delta_{0}>0$ such that

$$
\left(\frac{\gamma_{\delta}}{p^{\prime}}-\frac{1}{2}\right)>0, \quad \forall \delta \in\left[0, \delta_{0}\right]
$$

Thereby, by (2.10),

$$
d+1+\left|u_{n}\right|_{p^{\prime}} \geq\left(\frac{\gamma_{\delta}}{p^{\prime}}-\frac{1}{2}\right)\left|u_{n}\right|_{p^{\prime}}^{p^{\prime}}
$$

from where it follows that $\left(u_{n}\right)$ is a bounded sequence. As $L^{p^{\prime}}(\Omega)$ is a reflexive space, there is $u \in L^{p^{\prime}}(\Omega)$ such that $u_{n} \rightarrow u$ weakly in $L^{p^{\prime}}(\Omega)$. Then, by compactness of $K_{p^{\prime}, \Omega}$,

$$
K_{p^{\prime}, \Omega}\left(u_{n}\right) \rightarrow K_{p^{\prime}, \Omega}(u) \text { in } L^{p}(\Omega) \text { as } n \rightarrow \infty .
$$

On the other hand, the limit $J_{p^{\prime}, \delta}^{\prime}\left(u_{n}\right) \rightarrow 0$ in $\left(L^{p^{\prime}}(\Omega)\right)^{\prime}=L^{p}(\Omega)$ yields

$$
g_{p^{\prime}, \delta}\left(u_{n}\right)-K_{p^{\prime}, \Omega}\left(u_{n}\right) \rightarrow 0 \text { in } L^{p}(\Omega) .
$$

So, by (2.11),

$$
g_{p^{\prime}, \delta}\left(u_{n}\right) \rightarrow K_{p^{\prime}, \Omega}(u)=: w \text { in } L^{p}(\Omega) \text { as } n \rightarrow \infty .
$$

Then there is $h \in L^{p}(\Omega)$ such that

$$
\left|g_{p^{\prime}, \delta}\left(u_{n}(x)\right)\right| \leq h(x), \quad \forall n \in \mathbb{N},
$$




$$
g_{p^{\prime}, \delta}\left(u_{n}(x)\right) \rightarrow w(x) \text { a.e. in } \Omega .
$$

Let

$$
\Gamma:=\{x \in \Omega ;|w(x)|=a\} \text { and } \tilde{\Omega}:=\Omega \backslash \Gamma .
$$

We claim that $u_{n} \rightarrow f_{p, \delta}(w)$ in $L^{p^{\prime}}(\tilde{\Omega})$. If $x \in \tilde{\Omega}$, we have

$$
\left(f_{p, \delta} \circ g_{p^{\prime}, \delta}\right)\left(u_{n}(x)\right) \rightarrow f_{p, \delta}(w(x)),
$$

and also $\left|u_{n}(x)\right| \notin\left[a^{p-1},(1+\delta) a^{p-1}\right]$ for $n$ large enough, hence $\left(f_{p, \delta} \circ g_{p^{\prime}, \delta}\right)\left(u_{n}(x)\right)=u_{n}(x)$, so $u_{n}(x) \rightarrow f_{p, \delta}(w(x))$. Combining (2.13) and the fact that $f_{p, \delta}$ is odd and increasing, one easily derives a uniform estimate in $L^{p^{\prime}}(\tilde{\Omega})$ for sequence $\left(u_{n}\right)$, so by Dominated Convergence Theorem the conclusion follows.

On the other hand, by using the same type of arguments found [4, Theorem 1], it is possible to show that meas $(\Gamma)=0$. Then, the above analysis leads to

$$
u_{n} \rightarrow u \text { in } L^{p^{\prime}}(\Omega)
$$

and the proposition is proved.

We finish this section by proving that $J_{p^{\prime}, \delta}$ has a nontrivial critical point

Theorem 2.4 The mountain pass level of $J_{p^{\prime}, \delta}$, denoted by $c_{p^{\prime}, \delta}$, is a critical level.

Proof Propositions 2.2 and 2.3 permit to apply the Mountain Pass Theorem found in [1]. Then, there is a critical $u_{p^{\prime}, \delta} \in L^{p^{\prime}}(\Omega)$ whose the energy is equal to mountain pass level of $J_{p^{\prime}, \delta}$, that is,

$$
J_{p^{\prime}, \delta}^{\prime}\left(u_{p^{\prime}, \delta}\right)=0 \text { and } J_{p^{\prime}, \delta}\left(u_{p^{\prime}, \delta}\right)=c_{p^{\prime}, \delta}
$$

\section{Nehari manifold associated with $J_{p^{\prime}, \delta}$}

In this section, we will make a careful study of the Nehari manifold $\mathcal{N}_{p^{\prime}, \delta}$ associated with $J_{p^{\prime}, \delta}$ given by

$$
\begin{aligned}
\mathcal{N}_{p^{\prime}, \delta} & :=\left\{u \in L^{p^{\prime}}(\Omega) \backslash\{0\} ; J_{p^{\prime}, \delta}^{\prime}(u) u=0\right\} \\
& =\left\{u \in L^{p^{\prime}}(\Omega) \backslash\{0\} ; \int_{\Omega} g_{p^{\prime}, \delta}(u) u \mathrm{~d} x=\int_{\Omega} K_{p^{\prime}, \Omega}(u) u \mathrm{~d} x\right\} .
\end{aligned}
$$

It is worth pointing out that since $g_{p^{\prime}, \delta}$ is not a $C^{1}$ function, we cannot assert that $\mathcal{N}_{p^{\prime}, \delta}$ is a differentiable manifold. This fact brings for us some difficulties to apply Lagrange Multiplier on $\mathcal{N}_{p^{\prime}, \delta}$. However, we overcome this difficulty by adapting for our problem some arguments found in Szulkin and Weth [30].

Our first lemma follows by using the continuity of $K_{p^{\prime}, \Omega}$ together with the inequality $G_{p^{\prime}, \delta}(t)-\frac{1}{2} \operatorname{tg}_{p^{\prime}, \delta}(t) \geq C|t|^{p^{\prime}}$ for some constant $C>0$, and it has the following statement

Lemma 3.1 There is $\eta=\eta(p)>0$ such that

$$
|u|_{p^{\prime}}, J_{p^{\prime}, \delta}(u)>\eta, \quad \forall u \in \mathcal{N}_{p^{\prime}, \delta} .
$$


The second result can be obtained by using the same arguments found [32, Chapter 4], because the function $g_{p^{\prime}, \delta}$ is odd, $\frac{g_{p^{\prime}, \delta}(t)}{t}$ is decreasing for $t>0$ and $K_{p^{\prime}, \Omega}$ is a linear operator.

Lemma 3.2 For each $v \in L^{p^{\prime}}(\Omega) \backslash\{0\}$, there is an unique $t_{v}>0$ such that

$$
J_{p^{\prime}, \delta}^{\prime}\left(t_{v} v\right)\left(t_{v} v\right)=0 .
$$

Moreover,

$$
c_{p^{\prime}, \delta}=\inf _{u \in \mathcal{N}_{p^{\prime}, \delta}} J_{p^{\prime}, \delta}(u) .
$$

As an immediate consequence of the last lemma is the following corollary

Corollary 3.3 If $u$ is a critical point of $J_{p^{\prime}, \delta}$ with $u^{ \pm} \neq 0$, then $J_{p^{\prime}, \delta}(u) \geq 2 c_{p^{\prime}, \delta}$.

Proof The proof follows with the same type of arguments found in [10, Section 4] or [11, Theorem 2.4].

The next lemma is crucial in our approach, because it guarantees the continuity of the function $v \mapsto t_{v}$ in $L^{p^{\prime}}(\Omega) \backslash\{0\}$.

Lemma 3.4 For $\left(u_{n}\right) \subset L^{p^{\prime}}(\Omega)$ and $u \in L^{p^{\prime}}(\Omega) \backslash\{0\}$, let $t_{u_{n}}, t_{u}>0$ be as in (3.1). If $u_{n} \rightarrow u$ in $L^{p^{\prime}}(\Omega)$, then $t_{u_{n}} \rightarrow t_{u}$.

Proof For simplicity, set $t_{n}:=t_{u_{n}}$. First of all, note that $t_{n} \nrightarrow \nrightarrow 0$. Indeed, taking $v=u_{n}$ in (3.1) and using (2.2) and (2.9), we get

$$
\gamma_{\delta}\left|u_{n}\right|_{p^{\prime}}^{p^{\prime}} t_{n}^{p^{\prime}} \leq \int_{\Omega} g_{p^{\prime}, \delta}\left(t_{n} u_{n}\right) t_{n} u_{n} \mathrm{~d} x=\int_{\Omega} K_{p^{\prime}, \Omega}\left(t_{n} u_{n}\right)\left(t_{n} u_{n}\right) \mathrm{d} x \leq C t_{n}^{2}\left|u_{n}\right|_{p^{\prime}}^{2},
$$

for some $C>0$. So, for some $c>0$,

$$
c\left|u_{n}\right|_{p^{\prime}}^{p^{\prime}-2} \leq t_{n}^{2-p^{\prime}}
$$

and the desired property follows from the fact that $p^{\prime} \in(1,2)$ and $\left(u_{n}\right)$ are a bounded sequence in $L^{p^{\prime}}(\Omega)$.

Moreover, $\left(t_{n}\right)$ is bounded. In fact, the continuity of $K_{p^{\prime}, \Omega},(2.2)$ and (3.1) leads to $t_{n}^{p^{\prime}-2} C\left|u_{n}\right|_{p^{\prime}}^{p^{\prime}} \geq \frac{1}{t_{n}^{2}} \int_{\Omega} g_{p^{\prime}, \delta}\left(t_{n} u_{n}\right) t_{n} u_{n} \mathrm{~d} x=\int_{\Omega} K_{p^{\prime}, \Omega}\left(u_{n}\right)\left(u_{n}\right) \mathrm{d} x \rightarrow \int_{\Omega} K_{p^{\prime}, \Omega}(u) u \mathrm{~d} x>0$, which implies the boundedness of $\left(t_{n}\right)$.

Finally, up to a subsequence, we have $t_{n} \rightarrow t_{0}$. Then, by Lebesgue's Theorem,

$$
\begin{aligned}
\int_{\Omega} g_{p^{\prime}, \delta}\left(t_{0} u\right) t_{0} u \mathrm{~d} x & =\lim _{n} \int_{\Omega} g_{p^{\prime}, \delta}\left(t_{n} u_{n}\right) t_{n} u_{n} \mathrm{~d} x \\
& =\lim _{n} \int_{\Omega} K_{p^{\prime}, \Omega}\left(t_{n} u_{n}\right) t_{n} u_{n} \mathrm{~d} x=\int_{\Omega} K_{p^{\prime}, \Omega}\left(t_{0} u\right) t_{0} u \mathrm{~d} x .
\end{aligned}
$$

Now, the uniqueness of $t_{u}$ ensures that $t_{u}=t_{0}=\lim _{n \rightarrow+\infty} t_{n}$.

In the sequel, without loss of generality we assume that $0 \in \Omega$ and denote by $w_{p, r} \in$ $H_{0}^{1}\left(B_{r}(0)\right)$ be a positive ground-state solution of the problem

$$
\left\{\begin{array}{l}
-\Delta w=|w|^{p-2} w, \quad x \in B_{r}(0), \\
w=0, \quad x \in \partial B_{r}(0)
\end{array}\right.
$$


where $r>0$ is such that the sets

$$
\Omega^{+}:=\left\{x \in \mathbb{R}^{N} ; \operatorname{dist}(x, \Omega) \leq r\right\}, \quad \Omega^{-}:=\{x \in \Omega ; \operatorname{dist}(x, \partial \Omega) \geq r\}
$$

and $\Omega$ are homotopically equivalent. Hence,

$$
I_{p, B_{r}(0)}\left(w_{p, r}\right)=b_{p, B_{r}(0)} \text { and } I_{p, B_{r}(0)}^{\prime}\left(w_{p, r}\right)=0
$$

where

$$
I_{p, B_{r}(0)}(u)=\frac{1}{2} \int_{B_{r}(0)}|\nabla u|^{2} \mathrm{~d} x-\frac{1}{p} \int_{B_{r}(0)}|u|^{p} \mathrm{~d} x, \quad \forall u \in H_{0}^{1}\left(B_{r}(0)\right)
$$

and $b_{p, B_{r}(0)}$ denotes the mountain pass level associated with $I_{p, B_{r}(0)}$. It is well known that $w_{p, r}$ is radially symmetric and of class $C^{2}$. Therefore, $u_{p^{\prime}, r}:=w_{p, r}^{p-1}$ is positive, radially symmetric and a critical point of the functional $J_{p^{\prime}, B_{r}(0)}: L^{p^{\prime}}\left(B_{r}(0)\right) \rightarrow \mathbb{R}$ given by

$$
\begin{aligned}
J_{p^{\prime}, B_{r}(0)}(u) & =\int_{B_{r}(0)} G_{p^{\prime}}(u) \mathrm{d} x-\frac{1}{2} \int_{B_{r}(0)} K_{p^{\prime}, B_{r}(0)}(u) u \mathrm{~d} x \\
& =\frac{1}{p^{\prime}} \int_{B_{r}(0)}|u|^{p^{\prime}} \mathrm{d} x-\frac{1}{2} \int_{B_{r}(0)} K_{p^{\prime}, B_{r}(0)}(u) u \mathrm{~d} x .
\end{aligned}
$$

Moreover,

$$
\begin{aligned}
J_{p^{\prime}, B_{r}(0)}\left(u_{p^{\prime}, r}\right) & =\frac{1}{p^{\prime}} \int_{B_{r}(0)}\left|u_{p^{\prime}, r}\right|^{p^{\prime}} \mathrm{d} x-\frac{1}{2} \int_{B_{r}(0)} K_{p^{\prime}, B_{r}(0)}\left(u_{p^{\prime}, r}\right) u_{p^{\prime}, r} \mathrm{~d} x \\
& =\frac{1}{p^{\prime}} \int_{B_{r}(0)}\left|u_{p^{\prime}, r}\right|^{p^{\prime}} \mathrm{d} x-\frac{1}{2} \int_{B_{r}(0)}\left|u_{p^{\prime}, r}\right|^{p^{\prime}} \mathrm{d} x \\
& =\left(\frac{1}{p^{\prime}}-\frac{1}{2}\right) \int_{B_{r}(0)}\left|u_{p^{\prime}, r}\right|^{p^{\prime}} \mathrm{d} x=\left(\frac{1}{2}-\frac{1}{p}\right) \int_{B_{r}(0)}\left|u_{p^{\prime}, r}\right|^{p^{\prime}} \mathrm{d} x \\
& =\left(\frac{1}{2}-\frac{1}{p}\right) \int_{B_{r}(0)}\left|w_{p, r}\right|^{p} \mathrm{~d} x=I_{p, B_{r}(0)}\left(w_{p, r}\right)=b_{p, B_{r}(0)} .
\end{aligned}
$$

Arguing as in [3], it is possible to prove that $b_{p, B_{r}(0)}=c_{p^{\prime}, B_{r}(0)}$, where $c_{p^{\prime}, B_{r}(0)}$ denotes the mountain pass level of $J_{p^{\prime}, B_{r}(0)}$.

In the sequel, let $\Phi_{p^{\prime}, \delta}: \Omega^{-} \rightarrow \mathcal{N}_{p^{\prime}, \delta}$ be the map defined by

$$
\Phi_{p^{\prime}, \delta}(y)(x)= \begin{cases}t_{p^{\prime}, y} u_{p^{\prime}, r}(|x-y|), & x \in B_{r}(y), \\ 0, & x \in \Omega \backslash B_{r}(y),\end{cases}
$$

where $t_{p^{\prime}, y}>0$ is such that $t_{p^{\prime}, y} u_{p^{\prime}, r}(|.-y|) \in \mathcal{N}_{p^{\prime}, \delta}$, for each $y \in \Omega^{-}$. Using the function $\Phi_{p^{\prime}, \delta}(y)$, we are able to prove that

$$
c_{p^{\prime}, \delta} \leq c_{p^{\prime}, B_{r}(0)} \cdot
$$

Indeed, firstly it is very important to observe that from definition of $\Omega^{-}$and $r$, we have that $B_{r}(y) \subset \Omega$ for all $y \in \Omega^{-}$, consequently $\operatorname{supp}\left(\Phi_{p^{\prime}, \delta}(y)\right)=B_{r}(y) \subset \Omega$. Then,

$$
\begin{aligned}
c_{p^{\prime}, \delta} & \leq J_{p^{\prime}, \delta}\left(\Phi_{p^{\prime}, \delta}(y)\right)=J_{p^{\prime}, \delta}\left(t_{p^{\prime}, y} u_{p^{\prime}, r}\right) \\
& =\int_{\Omega} G_{p^{\prime}, \delta}\left(t_{p^{\prime}, y} u_{p^{\prime}, r}\right) \mathrm{d} x-\frac{t_{p^{\prime}, y}^{2}}{2} \int_{\Omega} K_{p^{\prime}, \Omega}\left(u_{p^{\prime}, r}\right) u_{p^{\prime}, r} \mathrm{~d} x .
\end{aligned}
$$


By the maximum principle,

$$
K_{p^{\prime}, \Omega}\left(u_{p^{\prime}, r}\right)>K_{p^{\prime}, B_{r}(0)}\left(u_{p^{\prime}, r}\right) \text { on } B_{r}(0),
$$

and so,

$$
\begin{aligned}
c_{p^{\prime}, \delta} & \leq J_{p^{\prime}, \delta}\left(\Phi_{p^{\prime}, \delta}(y)\right) \leq \int_{\Omega} G_{p^{\prime}, \delta}\left(t_{p^{\prime}, y} u_{p^{\prime}, r}\right) \mathrm{d} x-\frac{1}{2} \int_{\Omega} K_{p^{\prime}, \Omega}\left(t_{p^{\prime}, y} u_{p^{\prime}, r}\right) t_{p^{\prime}, y} u_{p^{\prime}, r} \mathrm{~d} x \\
& \leq \int_{B_{r}(0)} G_{p^{\prime}}\left(t_{p^{\prime}, y} u_{p^{\prime}, r}\right) \mathrm{d} x-\frac{1}{2} \int_{B_{r}(0)} K_{p^{\prime}, B_{r}(0)}\left(t_{p^{\prime}, y} u_{p^{\prime}, r}\right) t_{p^{\prime}, y} u_{p^{\prime}, r} \mathrm{~d} x \\
& =J_{p^{\prime}, B_{r}(0)}\left(t_{p^{\prime}, y} u_{p^{\prime}, r}\right) \leq J_{p^{\prime}, B_{r}(0)}\left(u_{p^{\prime}, r}\right)=c_{p^{\prime}, B_{r}(0)},
\end{aligned}
$$

which proves (3.3).

Our next result shows the behavior of the levels $c_{p^{\prime}, \delta}$ and $c_{p^{\prime}, B_{r}(0)}$ with respect to the numbers $p$ and $\delta$.

Proposition 3.5 The following limits hold:

$$
\lim _{p \rightarrow 2^{*}, \delta \rightarrow 0} c_{p^{\prime}, \delta}=\lim _{p \rightarrow 2^{*}} c_{p^{\prime}, B_{r}(0)}=c_{*}:=\frac{1}{N} S^{N / 2},
$$

where $S$ is the best constant for the embedding $H_{0}^{1}(\Omega) \hookrightarrow L^{2^{*}}(\Omega)$.

Proof We begin by showing the second limit. Let us denote by $I_{p}$ and $J_{p^{\prime}}$ the functionals $I_{p, 0}$ and $J_{p^{\prime}, 0}$, respectively. If $b_{p, B_{r}(0)}$ denotes the mountain pass level of $I_{p, B_{r}(0)}$, adapting the arguments found in [3], it follows $b_{p, B_{r}(0)}=c_{p^{\prime}, B_{r}(0)}$. Moreover, in [14] it was proved that

$$
b_{p, B_{r}(0)}=\left(\frac{1}{2}-\frac{1}{p}\right) m_{p, r}^{\frac{p}{p-2}}
$$

with

$$
m_{p, r}:=\inf _{w \in H_{0}^{1}\left(B_{r}(0)\right) \backslash\{0\}} \frac{\int_{B_{r}(0)}|\nabla w|^{2} \mathrm{~d} x}{\left(\int_{B_{r}(0)}|w|^{p} \mathrm{~d} x\right)^{2 / p}} \text { and } \lim _{p \rightarrow 2^{*}} m_{p, r}=S .
$$

Therefore,

$$
\lim _{p \rightarrow 2^{*}} c_{p^{\prime}, B_{r}(0)}=\lim _{p \rightarrow 2^{*}} b_{p, B_{r}(0)}=\lim _{p \rightarrow 2^{*}}\left(\frac{1}{2}-\frac{1}{p}\right) m_{p, r}^{\frac{p}{p-2}}=c_{*} .
$$

Here, we would like to point out that the above arguments could be made with $B_{r}(0)$ replaced by $\Omega$, because the result found in [14] still holds for $\Omega$. Then, if $c_{p^{\prime}}=c_{p^{\prime}, 0}$, we also have

$$
\lim _{p \rightarrow 2^{*}} c_{p^{\prime}}=c_{*} .
$$

Now, we deal with the first limit in the statement, that is,

$$
\lim _{p \rightarrow 2^{*}, \delta \rightarrow 0} c_{p^{\prime}, \delta}=c_{*} .
$$

Let $\left(\delta_{n}\right),\left(p_{n}\right)$ be sequences satisfy $\delta_{n} \rightarrow 0$ and $p_{n} \rightarrow 2^{*}$ as $n \rightarrow \infty$. By Theorem 2.4, for each $n \in \mathbb{N}$ there is $u_{n} \in L^{p_{n}^{\prime}}(\Omega)$ such that

$$
J_{p_{n}^{\prime}, \delta_{n}}\left(u_{n}\right)=c_{p_{n}^{\prime}, \delta_{n}} \text { and } J_{p_{n}^{\prime}, \delta_{n}}^{\prime}\left(u_{n}\right)=0 .
$$


Setting $t_{n}>0$ be such that $t_{n} u_{n} \in \mathcal{N}_{p_{n}^{\prime}}:=\mathcal{N}_{p_{n}^{\prime}, 0}$, we find

$$
c_{p_{n}^{\prime}} \leq J_{p_{n}^{\prime}}\left(t_{n} u_{n}\right)=J_{p_{n}^{\prime}, \delta_{n}}\left(t_{n} u_{n}\right)+\int_{\Omega}\left[G_{p_{n}^{\prime}}\left(t_{n} u_{n}\right)-G_{p_{n}^{\prime}, \delta_{n}}\left(t_{n} u_{n}\right)\right] \mathrm{d} x .
$$

\section{Claim 3.6}

$$
\int_{\Omega}\left[G_{p_{n}^{\prime}}\left(t_{n} u_{n}\right)-G_{p_{n}^{\prime}, \delta_{n}}\left(t_{n} u_{n}\right)\right] \mathrm{d} x=o_{n}(1) .
$$

Indeed, by the definitions of $G_{p^{\prime}, \delta}$ and $G_{p^{\prime}}$,

$$
0 \leq G_{p^{\prime}}(s)-G_{p^{\prime}, \delta}(s) \leq\left(1-\gamma_{\delta}\right) \frac{1}{p^{\prime}}|s|^{p^{\prime}}, \quad \forall s \in \mathbb{R},
$$

and so,

$$
0 \leq \int_{\Omega}\left[G_{p_{n}^{\prime}}\left(t_{n} u_{n}\right)-G_{p_{n}^{\prime}, \delta_{n}}\left(t_{n} u_{n}\right)\right] \mathrm{d} x \leq\left(1-\gamma_{\delta_{n}}\right) \frac{1}{p_{n}^{\prime}} t_{n}^{p_{n}^{\prime}} \int_{\Omega}\left|u_{n}\right|^{p_{n}^{\prime}} \mathrm{d} x .
$$

From this, we will get the desired conclusion by showing that $\left(\left|t_{n} u_{n}\right|_{p_{n}^{\prime}}\right)$ is bounded, since $\gamma_{\delta_{n}} \rightarrow 1$ as $n \rightarrow \infty$. Firstly, from inequality

$$
G_{p^{\prime}, 0}(t) \geq G_{p^{\prime}, \delta}(t), \quad \forall t \in \mathbb{R},
$$

we have that

$$
J_{p^{\prime}, 0}(u) \geq J_{p^{\prime}, \delta}(u) \quad \forall u \in L^{p^{\prime}}(\Omega),
$$

implying that $c_{p_{n}^{\prime}} \geq c_{p_{n}^{\prime}, \delta_{n}}$ for all $n \in \mathbb{N}$. From this, $\left(\left|u_{n}\right|_{p_{n}^{\prime}}\right)$ is bounded, because $\left(c_{p_{n}^{\prime}}\right)$ is a bounded sequence and

$$
\begin{aligned}
c_{p_{n}^{\prime}} & \geq c_{p_{n}^{\prime}, \delta_{n}}=J_{p_{n}^{\prime}, \delta_{n}}\left(u_{n}\right)=J_{p_{n}^{\prime}, \delta_{n}}\left(u_{n}\right)-\frac{1}{2} J_{p_{n}^{\prime}, \delta_{n}}^{\prime}\left(u_{n}\right)\left(u_{n}\right) \\
& =\int_{\Omega}\left[G_{p_{n}^{\prime}, \delta_{n}}\left(u_{n}\right)-\frac{1}{2} g_{p_{n}^{\prime}, \delta_{n}}\left(u_{n}\right) u_{n}\right] \mathrm{d} x \geq\left(\frac{\gamma_{\delta_{n}}}{p_{n}^{\prime}}-\frac{1}{2}\right) \int_{\Omega}\left|u_{n}\right|^{p^{\prime}} \mathrm{d} x .
\end{aligned}
$$

Next, we will work to show that $\left(t_{n}\right)$ is also a bounded sequence. To this end, we need to prove that

$$
\liminf _{n \rightarrow \infty}\left|u_{n}\right|_{p_{n}^{\prime}}>0 .
$$

As $p_{n}^{\prime}>2^{+}$and $|\Omega|<\infty$, it follows that

$$
K_{p_{n}^{\prime}, \Omega}\left(u_{n}\right)=K_{2^{+}, \Omega}\left(u_{n}\right), \forall n \in \mathbb{N} .
$$

As $u_{n} \in \mathcal{N}_{p_{n}^{\prime}, \delta_{n}}$, the above equality combined with Hölder inequality gives

$$
\gamma_{\delta}\left|u_{n}\right|_{p_{n}^{\prime}}^{p_{n}^{\prime}} \leq \int_{\Omega} g_{p_{n}^{\prime}, \delta_{n}}\left(u_{n}\right) u_{n} \mathrm{~d} x=\int_{\Omega} K_{2^{+}, \Omega}\left(u_{n}\right) u_{n} \mathrm{~d} x \leq C\left|u_{n}\right|_{2^{+}}^{2} \leq C|\Omega|^{\frac{2}{\theta_{n}}}\left|u_{n}\right|_{p_{n}^{\prime}}^{2},
$$

where $\frac{1}{2^{+}}=\frac{1}{p_{n}^{\prime}}+\frac{1}{\theta_{n}}$. Then,

$$
1 \leq C|\Omega|^{\frac{2}{\theta_{n}}}\left|u_{n}\right|_{p_{n}^{\prime}}^{2-p_{n}^{\prime}}
$$

Once $\theta_{n} \rightarrow \infty$ and $p_{n}^{\prime} \rightarrow 2^{+}$as $p_{n} \rightarrow 2^{*}$, the last inequality implies that there is $\kappa>0$ such that $\left|u_{n}\right|_{p_{n}^{\prime}}>\kappa$ for $n$ large enough, which proves (3.10). 
Now, by using the fact $u_{n} \in \mathcal{N}_{p_{n}^{\prime}, \delta_{n}}$ together with (2.2) and (3.10), we obtain

$$
\int_{\Omega} K_{p_{n}^{\prime}, \Omega}\left(u_{n}\right) u_{n} \mathrm{~d} x=\int_{\Omega} g_{p_{n}^{\prime}, \delta_{n}}\left(u_{n}\right) u_{n} \mathrm{~d} x \geq \gamma \delta_{n} \int_{\Omega}\left|u_{n}\right|^{p_{n}^{\prime}} \mathrm{d} x>\kappa,
$$

for $n$ large enough. Hence,

$$
\frac{c_{*}}{2} \leq c_{p_{n}^{\prime}} \leq J_{p_{n}^{\prime}}\left(t_{n} u_{n}\right)=\frac{t_{n}^{p_{n}^{\prime}}}{p_{n}^{\prime}} \int_{\Omega}\left|u_{n}\right|^{p_{n}^{\prime}} \mathrm{d} x-\frac{t_{n}^{2}}{2} \int_{\Omega} K_{p_{n}^{\prime}, \Omega}\left(u_{n}\right) u_{n} \mathrm{~d} x,
$$

for $n$ large enough. Gathering the boundedness of $\left(\left|u_{n}\right|_{p_{n}^{\prime}}\right)$ with (3.11), we derive

$$
\frac{c_{*}}{2} \leq c t_{n}^{p_{n}^{\prime}}-\tau t_{n}^{2}, \forall n \in \mathbb{N},
$$

from where it follows that $\left(t_{n}\right)$ is bounded. From this, $\left(\left|t_{n} u_{n}\right|_{p_{n}^{\prime}}\right)$ is bounded, which finishes the proof of Claim 3.6. Therefore from (3.3) and (3.7)

$$
c_{p_{n}^{\prime}} \leq c_{p_{n}^{\prime}, \delta_{n}}+o_{n}(1) \leq c_{p_{n}^{\prime}}+o_{n}(1) .
$$

As $\left(p_{n}\right)$ and $\left(\delta_{n}\right)$ are arbitrary sequences, (3.5) gives

$$
\lim _{p \rightarrow 2^{*}, \delta \rightarrow 0} c_{p^{\prime}, \delta}=c_{*} .
$$

The next step would be to determine whether or not the restriction of $J_{p^{\prime}, \delta}$ to $\mathcal{N}_{p^{\prime}, \delta}$ satisfies the $(P S)$-condition. The standard approach would lead us to the study of the second derivative of $J_{p^{\prime}, \delta}$, which we cannot compute, since this functional is not twice differentiable. With this in mind, we will adapt for our case some ideas explored in [30].

Consider the application

$$
\hat{m}_{p^{\prime}, \delta}: L^{p^{\prime}}(\Omega) \backslash\{0\} \rightarrow \mathcal{N}_{p^{\prime}, \delta}
$$

given by

$$
\hat{m}_{p^{\prime}, \delta}(u)=t_{u} u,
$$

where $t_{u}$ is defined by (3.1). Using the above notations, it is possible to prove that

(a) $\hat{m}_{p^{\prime}, \delta}$ is a continuous application.

(b) There is $\tau>0$ such that $t_{u}>\tau, \forall u \in \mathcal{S}_{p^{\prime}}=\left\{u \in L^{p^{\prime}}(\Omega):|u|_{p^{\prime}}=1\right\}$;

Indeed, if $\left(u_{n}\right) \subset L^{p^{\prime}}(\Omega)$ is such that $t_{u_{n}} \rightarrow 0$ as $n \rightarrow \infty$, then $t_{u_{n}} u_{n} \rightarrow 0$ as $n \rightarrow \infty$.

This contradicts Lemma 3.1, since $t_{u_{n}} u_{n} \in \mathcal{N}_{p^{\prime}, \delta}$.

(c) Given $\mathcal{W} \subset \mathcal{S}_{p^{\prime}}$ compact, there is $C_{\mathcal{W}}>0$ such that $C_{\mathcal{W}}>t_{u}, \forall u \in \mathcal{W}$;

In fact, this is a consequence of the continuity of the application $v \mapsto t_{v}$, as shown in Lemma 3.4.

In the sequel, we consider the application $m_{p^{\prime}, \delta}: \mathcal{S}_{p^{\prime}} \rightarrow \mathcal{N}_{p^{\prime}, \delta}$, the restriction of $\hat{m}_{p^{\prime}, \delta}$ to the sphere $\mathcal{S}_{p^{\prime}}$. Observe that $m_{p^{\prime}, \delta}$ is a homeomorphism, with inverse given by

$$
m_{p^{\prime}, \delta}^{-1}(u)=\frac{u}{|u|_{p^{\prime}}}, \quad \forall u \in \mathcal{N}_{p^{\prime}, \delta}
$$

Let us also consider the application $\hat{\Psi}_{p^{\prime}, \delta}: L^{p^{\prime}}(\Omega) \backslash\{0\} \rightarrow \mathbb{R}$ given by

$$
\hat{\Psi}_{p^{\prime}, \delta}(u):=J_{p^{\prime}, \delta}\left(\hat{m}_{p^{\prime}, \delta}(u)\right),
$$


and its restriction to the sphere, $\Psi_{p^{\prime}, \delta}: \mathcal{S}_{p^{\prime}} \rightarrow \mathbb{R}$. Note that both $\hat{\Psi}_{p^{\prime}, \delta}$ and $\Psi_{p^{\prime}, \delta}$ are continuous. The following result is crucial in our approach and its proof can be found in [30, Chapter 3].

Lemma 3.7 The applications defined above satisfy:

(i) $\hat{\Psi}_{p^{\prime}, \delta} \in C^{1}\left(L^{p^{\prime}}(\Omega) \backslash\{0\}, \mathbb{R}\right)$ and, for $u \in L^{p^{\prime}}(\Omega) \backslash\{0\}$,

$$
\begin{aligned}
\hat{\Psi}_{p^{\prime}, \delta}^{\prime}(u) v & =\frac{\left|\hat{m}_{p^{\prime}, \delta}(u)\right|_{p^{\prime}}}{|u|_{p^{\prime}}} J_{p^{\prime}, \delta}^{\prime}\left(\hat{m}_{p^{\prime}, \delta}(u)\right) v, \\
& =t_{u} J_{p^{\prime}, \delta}^{\prime}\left(\hat{m}_{p^{\prime}, \delta}(u)\right) v, \quad \forall v \in L^{p^{\prime}}(\Omega) ;
\end{aligned}
$$

(ii) $\Psi_{p^{\prime}, \delta} \in C^{1}\left(\mathcal{S}_{p^{\prime}}, \mathbb{R}\right)$ and, for $u \in \mathcal{S}_{p^{\prime}}$,

$$
\Psi_{p^{\prime}, \delta}^{\prime}(u) v=\left|m_{p^{\prime}, \delta}(u)\right|_{p^{\prime}} J_{p^{\prime}, \delta}^{\prime}\left(m_{p^{\prime}, \delta}(u)\right) v, \quad \forall v \in T_{u} \mathcal{S}_{p^{\prime}},
$$

where $T_{u} \mathcal{S}_{p^{\prime}}$ denotes the tangent space of $\mathcal{S}_{p^{\prime}}$ at $u$;

(iii) If $\left(u_{n}\right) \subset \mathcal{S}_{p^{\prime}}$ is a (PS) sequence for $\Psi_{p^{\prime}, \delta}$, then $\left(m_{p^{\prime}, \delta}\left(u_{n}\right)\right)$ is a $(P S)$ sequence for $J_{p^{\prime}, \delta}$; if $\left(u_{n}\right) \subset \mathcal{N}_{p^{\prime}, \delta}$ is a (bounded) $(P S)$ sequence for $J_{p^{\prime}, \delta}$, then $\left(m_{p^{\prime}, \delta}^{-1}\left(u_{n}\right)\right)$ is a $(P S)$ sequence for $\Psi_{p^{\prime}, \delta}$;

(iv) $u \in \mathcal{S}_{p^{\prime}}$ is a critical point of $\Psi_{p^{\prime}, \delta}$ if, and only if, $m_{p^{\prime}, \delta}(u)$ is a (nonzero) critical point of $J_{p^{\prime}, \delta}$. Moreover,

$$
\inf _{\mathcal{S}_{p^{\prime}}} \Psi_{p^{\prime}, \delta}=\inf _{\mathcal{N}_{p^{\prime}, \delta}} J_{p^{\prime}, \delta}
$$

Corollary $3.8 \Psi_{p^{\prime}, \delta}$ is bounded from below and satisfies the (PS) condition.

Proof The boundedness follows by a combination of the previous result and Lemma 3.1. On the $(P S)$ condition, let $\left(u_{n}\right) \subset \mathcal{S}_{p^{\prime}}$ be a $(P S)$ sequence for $\Psi_{p^{\prime}, \delta}$. Thus, by Lemma 3.7-(iii), $\left(m_{p^{\prime}, \delta}\left(u_{n}\right)\right)$ is a $(P S)$ sequence for $J_{p^{\prime}, \delta}$. By Proposition 2.3, $\left(m_{p^{\prime}, \delta}\left(u_{n}\right)\right)$ has a strongly convergent subsequence. Since $m_{p^{\prime}, \delta}$ is a homeomorphism, $\left(u_{n}\right)$ has a strongly convergent subsequence, that is, $\Psi_{p^{\prime}, \delta}$ satisfies the ( $P S$ ) condition.

Before concluding this section, we will show that Palais-Smale sequence of $J_{2+}$ produces a Palais-Smale sequence for $I_{2^{*}}: H_{0}^{1}(\Omega) \rightarrow \mathbb{R}$ given by

$$
I_{2 *}(u)=\frac{1}{2} \int_{\Omega}|\nabla u|^{2} \mathrm{~d} x-\frac{1}{2^{*}} \int_{\Omega}|u|^{2^{*}} \mathrm{~d} x .
$$

Lemma 3.9 If $\left(u_{n}\right) \subset \mathcal{N}_{2}$ is a $(P S)_{d}$ sequence of $J_{2^{+}}$, then there is $t_{n}>0$ such that $v_{n}=t_{n}\left|u_{n}\right|^{2^{+}-2} u_{n} \in \mathcal{M}_{2 *}$, where $\mathcal{M}_{2 *}$ is the Nehari manifold associated with $I_{2 *}$. Moreover, the sequence $\left(v_{n}\right)$ is a $(P S)_{d}$ sequence of $I_{2^{*}}$.

Proof Let $\left(u_{n}\right) \subset \mathcal{N}_{2^{+}}$be a $(P S)_{d}$ sequence for $J_{2^{+}}$, that is, $\left(u_{n}\right)$ satisfies

$$
J_{2^{+}}^{\prime}\left(u_{n}\right) u_{n}=0, \quad J_{2^{+}}\left(u_{n}\right)=d+o_{n}(1) \text { and }\left\|J_{2^{+}}^{\prime}\left(u_{n}\right)\right\|_{L^{2^{*}}(\Omega)}=o_{n}(1) .
$$

Then $\left(u_{n}\right) \subset L^{2^{+}}(\Omega)$ is a bounded sequence. In what follows, we define $\left(w_{n}\right) \subset H_{0}^{1}(\Omega)$ and $\left(v_{n}\right) \subset L^{2^{*}}(\Omega)$ by $w_{n}:=K_{2^{+}, \Omega}\left(u_{n}\right)$ and $\tilde{v}_{n}:=\left|u_{n}\right|^{2^{+}-2} u_{n}$, for each $n \in \mathbb{N}$. So $u_{n}=\left|\tilde{v}_{n}\right|^{2^{*}-2} \tilde{v}_{n}$ and $w_{n}$ is the unique solution of the problem

$$
\left\{\begin{array}{l}
-\Delta w_{n}=u_{n}, \quad x \in \Omega, \\
w_{n}=0, \quad \text { on } \partial \Omega
\end{array}\right.
$$


By (3.12) and the definitions of $w_{n}$ and $v_{n}$, both $\left(w_{n}\right),\left(\tilde{v}_{n}\right)$ are bounded sequences with $\left|\tilde{v}_{n}-w_{n}\right|_{2^{*}} \rightarrow 0$ as $n \rightarrow \infty$, that is, $w_{n}=\tilde{v}_{n}+o_{n}(1)$ in $L^{2^{*}}(\Omega)$.

The sequence $\left(w_{n}\right)$ is a $(P S)_{d}$ sequence for $I_{2 *}$. Indeed, for any $\phi \in H_{0}^{1}(\Omega),(3.13)$ gives

$$
\begin{aligned}
I_{2^{*}}^{\prime}\left(w_{n}\right) \phi & =\int_{\Omega} \nabla w_{n} \nabla \phi \mathrm{d} x-\int_{\Omega}\left|w_{n}\right|^{2^{*}-2} w_{n} \phi \mathrm{d} x \\
& =\int_{\Omega} u_{n} \phi \mathrm{d} x-\int_{\Omega}\left|w_{n}\right|^{2^{*}-2} w_{n} \phi \mathrm{d} x \\
& =\int_{\Omega}\left(\left|\tilde{v}_{n}\right|^{2^{*}-2} \tilde{v}_{n}-\left|w_{n}\right|^{2^{*}-2} w_{n}\right) \phi \mathrm{d} x \\
& \leq\left. C|| \tilde{v}_{n}\right|^{2^{*}-2} \tilde{v}_{n}-\left.\left|w_{n}\right|^{2^{*}-2} w_{n}\right|_{2^{+}}\|\phi\|
\end{aligned}
$$

and so,

$$
\left\|I_{2^{*}}^{\prime}\left(u_{n}\right)\right\|_{H^{-1}(\Omega)} \leq\left. C|| \tilde{v}_{n}\right|^{2^{*}-2} \tilde{v}_{n}-\left.\left|w_{n}\right|^{2^{*}-2} w_{n}\right|_{2^{+}}
$$

As $w_{n}-\tilde{v}_{n} \rightarrow 0$ in $L^{2^{*}}(\Omega)$, it follows that

$$
\left\|I_{2^{*}}^{\prime}\left(w_{n}\right)\right\|_{H^{-1}(\Omega)}=o_{n}(1) .
$$

Moreover, since $J_{2^{+}}^{\prime}\left(u_{n}\right) u_{n}=0$,

$$
\begin{aligned}
I_{2^{*}}\left(w_{n}\right) & =\frac{1}{2} \int_{\Omega}\left|\nabla w_{n}\right|^{2} \mathrm{~d} x-\frac{1}{2^{*}} \int_{\Omega}\left|w_{n}\right|^{2^{*}} \mathrm{~d} x \\
& =\frac{1}{2} \int_{\Omega} w_{n} u_{n} \mathrm{~d} x-\frac{1}{2^{*}} \int_{\Omega}\left|v_{n}\right|^{2^{*}} \mathrm{~d} x+o_{n}(1) \\
& =\frac{1}{2} \int_{\Omega} w_{n} u_{n} \mathrm{~d} x-\frac{1}{2^{*}} \int_{\Omega}\left|u_{n}\right|^{2^{+}} \mathrm{d} x+o_{n}(1) \\
& =\left(\frac{1}{2}-\frac{1}{2^{*}}\right) \int_{\Omega}\left|u_{n}\right|^{2^{+}} \mathrm{d} x+o_{n}(1) \\
& =\left(\frac{1}{2^{+}}-\frac{1}{2}\right) \int_{\Omega}\left|u_{n}\right|^{2^{+}} \mathrm{d} x+o_{n}(1) \\
& =J_{2^{+}}\left(u_{n}\right)+o_{n}(1)=d+o_{n}(1) .
\end{aligned}
$$

Thus, $\left(w_{n}\right)$ is a $(P S)_{d}$ sequence for $I_{2^{*}}$. Next, fix $\tilde{w}_{n}:=t_{n} w_{n}$ where $t_{n}>0$ is such that $t_{n} w_{n} \in \mathcal{M}_{2^{*}}$. We claim that $\left(\tilde{w}_{n}\right)$ is a $(P S)_{d}$ for $I_{2^{*}}$. Indeed, once $\left(u_{n}\right)$ is a bounded sequence on $\mathcal{N}_{2^{+}}$, then $\liminf _{n \rightarrow \infty}\left|u_{n}\right|_{2^{+}}>0$. Furthermore, using again that $w_{n}=v_{n}+o_{n}(1)$ in $L^{2^{*}}(\Omega)$, we see that $t_{n}$ satisfies

$$
\begin{aligned}
t_{n}^{2} \int_{\Omega}\left|\nabla w_{n}\right|^{2} \mathrm{~d} x & =t_{n}^{2^{*}} \int_{\Omega}\left|w_{n}\right|^{2^{*}} \mathrm{~d} x \\
& =t_{n}^{2^{*}}\left[\int_{\Omega}\left|v_{n}\right|^{2^{*}} \mathrm{~d} x+o_{n}(1)\right] \\
& =t_{n}^{2^{*}} \int_{\Omega}\left|u_{n}\right|^{2^{+}} \mathrm{d} x+t_{n}^{2^{*}} o_{n}(1),
\end{aligned}
$$

which leads to

$$
\left(t_{n}^{2-2^{*}}-1\right) \int_{\Omega}\left|u_{n}\right|^{2^{+}} \mathrm{d} x=o_{n}(1) .
$$


Therefore, as $\left(u_{n}\right)$ does not vanish, $t_{n} \rightarrow 1$ as $n \rightarrow \infty$ which permits to conclude that $\left(\tilde{w}_{n}\right)$ is a $(P S)_{d}$ sequence for $I_{2^{*}}$. Hence, the sequence $\left(v_{n}\right)$ given by $v_{n}=t_{n} \tilde{v}_{n}=t_{n}\left|u_{n}\right|^{2^{+}-2} u_{n}$ is also a $(P S)_{d}$ sequence for $I_{2^{*}}$.

\section{Proof of Theorem 1.1}

After the study made in the previous section, we are able to prove our main result. To this end, we will consider the application $\beta: \mathcal{N}_{p^{\prime}, \delta} \rightarrow \mathbb{R}^{N}$ given by

$$
\beta(u)=\frac{\int_{\Omega} x|u|^{2^{+}} \mathrm{d} x}{\int_{\Omega}|u|^{2^{+}} \mathrm{d} x} .
$$

Once $L^{p^{\prime}}(\Omega) \hookrightarrow L^{2^{+}}(\Omega), \beta$ is well defined and

$$
\beta \circ \Phi_{p^{\prime}, \delta}(x)=x, \quad \forall x \in \Omega^{-} .
$$

The next result establishes an important estimate associated with $\beta$.

Proposition 4.1 There are $\epsilon, p^{*}, \delta_{1}>0$ such that for each $p \in\left(p^{*}, 2^{*}\right)$ and $\delta \in\left(0, \delta_{1}\right)$, if $u \in \mathcal{N}_{p^{\prime}, \delta}$ satisfies $J_{p^{\prime}, \delta}(u) \leq c_{*}+\epsilon$, then $\beta(u) \in \Omega^{+}$, where $c_{*}$ is defined in Proposition 3.5 .

Suppose by contradiction that the result is false. Then, there are sequences $\left(\epsilon_{n}\right),\left(p_{n}\right),\left(\delta_{n}\right)$ with $\epsilon_{n} \rightarrow 0, \delta_{n} \rightarrow 0, p_{n} \rightarrow 2^{*}$ and $u_{n} \in \mathcal{N}_{p_{n}^{\prime}, \delta_{n}}$ such that

$$
J_{p_{n}^{\prime}, \delta_{n}}\left(u_{n}\right) \leq c_{*}+\epsilon_{n} \text { and } \beta\left(u_{n}\right) \notin \Omega^{+} \text {. }
$$

To simplify the notation, we will use $J_{n}:=J_{p_{n}^{\prime}, \delta_{n}}, \mathcal{N}_{n}:=\mathcal{N}_{p_{n}^{\prime}, \delta_{n}}, G_{n}:=G_{p_{n}^{\prime}, \delta_{n}}, g_{n}:=g_{p_{n}^{\prime}, \delta_{n}}$ and $K_{n}:=K_{p_{n}^{\prime}, \Omega \text {. }}$

We begin noticing that $\left(\left|u_{n}\right|_{p_{n}^{\prime}}\right)$ is bounded, since (2.2) and (2.3) lead to

$$
\begin{aligned}
c_{*}+\epsilon_{n} & \geq J_{n}\left(u_{n}\right)=J_{n}\left(u_{n}\right)-\frac{1}{2} J_{n}^{\prime}\left(u_{n}\right) u_{n} \\
& =\int_{\Omega}\left(G_{n}\left(u_{n}\right)-\frac{1}{2} g_{n}\left(u_{n}\right) u_{n}\right) \mathrm{d} x \\
& \geq\left(\frac{\gamma_{\delta_{n}}}{p_{n}^{\prime}}-\frac{1}{2}\right)\left|u_{n}\right|_{p_{n}^{\prime}}^{p_{n}^{\prime}},
\end{aligned}
$$

and $\left(\frac{\gamma \delta_{n}}{p_{n}^{\prime}}-\frac{1}{2}\right) \rightarrow \frac{1}{N}$ as $n \rightarrow \infty$.

Claim 4.2 There is $t_{n}>0$ such that $t_{n} u_{n} \in \mathcal{N}_{p_{n}^{\prime}}$, that is, $J_{p_{n}^{\prime}}^{\prime}\left(t_{n} u_{n}\right) t_{n} u_{n}=0$, and $t_{n} \rightarrow 1$ as $n \rightarrow \infty$.

The existence of $\operatorname{such}\left(t_{n}\right)$ is a consequence of the definition of $J_{p_{n}^{\prime}}$. Thus, for each $n \in \mathbb{N}$,

$$
\int_{\Omega}\left|t_{n} u_{n}\right|^{p_{n}^{\prime}} \mathrm{d} x=\int_{\Omega} K_{n}\left(t_{n} u_{n}\right) t_{n} u_{n} \mathrm{~d} x,
$$

that is,

$$
t_{n}^{p_{n}^{\prime}-2} \int_{\Omega}\left|u_{n}\right|^{p_{n}^{\prime}} \mathrm{d} x=\int_{\Omega} K_{n}\left(u_{n}\right) u_{n} \mathrm{~d} x .
$$


Since $u_{n} \in \mathcal{N}_{n},(2.2)$ gives

$$
\int_{\Omega} K_{n}\left(u_{n}\right) u_{n} \mathrm{~d} x=\int_{\Omega} g_{n}\left(u_{n}\right) u_{n} \mathrm{~d} x=\int_{\Omega}\left|u_{n}\right|^{p_{n}^{\prime}} \mathrm{d} x+o_{n}(1) .
$$

By (4.3) and (4.4),

$$
\left(t_{n}^{p_{n}^{\prime}-2}-1\right) \int_{\Omega}\left|u_{n}\right|^{p_{n}^{\prime}} \mathrm{d} x=o_{n}(1)
$$

Moreover, by (2.3),

$$
\begin{aligned}
c_{p_{n}^{\prime}, \delta_{n}} & \leq J_{n}\left(u_{n}\right)=\int_{\Omega}\left(G_{n}\left(u_{n}\right)-\frac{1}{2} K_{n}\left(u_{n}\right) u_{n}\right) \mathrm{d} x \\
& =\int_{\Omega}\left(G_{n}\left(u_{n}\right)-\frac{1}{2} g_{n}\left(u_{n}\right) u_{n}\right) \mathrm{d} x \\
& \leq \frac{1}{p_{n}^{\prime}} \int_{\Omega}\left|u_{n}\right|^{p_{n}^{\prime}} \mathrm{d} x .
\end{aligned}
$$

Then, by Proposition 3.5, $\liminf _{n \rightarrow \infty}\left|u_{n}\right|_{p_{n}^{\prime}}^{p_{n}^{\prime}}>0$. Thereby, (4.5) ensures that $\lim _{n \rightarrow \infty} t_{n}=1$ and the claim is proved.

Let $\tilde{u}_{n}:=t_{n} u_{n}$, for all $n \in \mathbb{N}$. Since $\tilde{u}_{n} \in \mathcal{N}_{p_{n}^{\prime}}$, by using (4.2) and the same argument explored in the proof of Claim 3.6, we get

$$
\begin{aligned}
c_{*}+o_{n}(1) & =c_{p_{n}^{\prime}} \leq J_{p_{n}^{\prime}}\left(\tilde{u}_{n}\right)=J_{p_{n}^{\prime}}\left(t_{n} u_{n}\right) \\
& \leq J_{n}\left(u_{n}\right)+o_{n}(1) \leq c_{*}+\epsilon_{n}+o_{n}(1),
\end{aligned}
$$

that is,

$$
\lim _{n \rightarrow \infty} J_{p_{n}^{\prime}}\left(\tilde{u}_{n}\right)=c_{*} .
$$

Now observe that by the definition of $J_{2^{+}}$, there is $r_{n}>0$ such that $r_{n} \tilde{u}_{n} \in \mathcal{N}_{2^{+}}$.

Claim $4.3\left(\left|\tilde{u}_{n}\right|_{p_{n}^{\prime}}\right)$ and $\left(r_{n}\right)$ are bounded sequences and $\liminf _{n \rightarrow \infty}\left|r_{n} \tilde{u}_{n}\right|_{p_{n}^{\prime}}^{p_{n}^{\prime}}>0$.

In fact, once $r_{n} \tilde{u}_{n} \in \mathcal{N}_{2^{+}}$,

$$
\int_{\Omega}\left|r_{n} \tilde{u}_{n}\right|^{2^{+}} \mathrm{d} x=\int_{\Omega} K_{2^{+}, \Omega}\left(r_{n} \tilde{u}_{n}\right) r_{n} \tilde{u}_{n} \mathrm{~d} x
$$

that is,

$$
\frac{1}{r_{n}^{2-2^{+}}} \int_{\Omega}\left|\tilde{u}_{n}\right|^{2^{+}} \mathrm{d} x=\int_{\Omega} K_{2^{+}, \Omega}\left(\tilde{u}_{n}\right) \tilde{u}_{n} \mathrm{~d} x
$$

from where it follows that

$$
\frac{1}{r_{n}^{2-2^{+}}} \int_{\Omega}\left|\tilde{u}_{n}\right|^{2^{+}} \mathrm{d} x=\int_{\Omega} K_{p_{n}^{\prime}, \Omega}\left(\tilde{u}_{n}\right) \tilde{u}_{n} \mathrm{~d} x .
$$

Here, we had used the fact that $K_{p_{n}^{\prime}, \Omega}\left(\tilde{u}_{n}\right)=K_{2^{*}, \Omega}\left(\tilde{u}_{n}\right)$, because $p_{n}^{\prime}>2^{+}$and meas $(\Omega)<$ $\infty$.

Besides, since $\tilde{u}_{n} \in \mathcal{N}_{p_{n}^{\prime}}$ and $J_{p_{n}^{\prime}}\left(\tilde{u}_{n}\right) \rightarrow c_{*}$,

$$
\left(\frac{1}{2}-\frac{1}{p_{n}^{\prime}}\right) \int_{\Omega}\left|\tilde{u}_{n}\right|^{p_{n}^{\prime}} \mathrm{d} x=\left(\frac{1}{p_{n}}-\frac{1}{2}\right) \int_{\Omega}\left|\tilde{u}_{n}\right|^{p_{n}^{\prime}} \mathrm{d} x=J_{p_{n}^{\prime}}\left(\tilde{u}_{n}\right)=c_{*}+o_{n}(1),
$$


so

$$
\lim _{n \rightarrow \infty} \int_{\Omega}\left|\tilde{u}_{n}\right|^{p_{n}^{\prime}} \mathrm{d} x=N c_{*}=S^{N / 2}
$$

Since $\tilde{u}_{n}=t_{n} u_{n}$ and $t_{n} \rightarrow 1$, it follows that $\left(\left|u_{n}\right|_{p_{n}^{\prime}}\right)$ is also bounded. Using Hölder inequality,

$$
\int_{\Omega}\left|\tilde{u}_{n}\right|^{2^{+}} \mathrm{d} x \leq|\Omega|^{\frac{2^{+}}{\theta_{n}}}\left(\int_{\Omega}\left|u_{n}\right|^{p_{n}^{\prime}} \mathrm{d} x\right)^{\frac{2^{+}}{p_{n}^{\prime}}},
$$

where $\frac{1}{\theta_{n}}=\frac{1}{2^{+}}-\frac{1}{p_{n}^{\prime}} \rightarrow 0$ as $n \rightarrow \infty$. Therefore,

$$
\int_{\Omega}\left|\tilde{u}_{n}\right|^{2^{+}} \mathrm{d} x \leq o_{n}(1)+\left(\int_{\Omega}\left|\tilde{u}_{n}\right|^{p_{n}^{\prime}} \mathrm{d} x\right)^{\frac{2^{+}}{p_{n}^{\prime}}},
$$

and $\left(\left|\tilde{u}_{n}\right|_{2^{+}}\right)$is bounded. Moreover, arguing as in the proof of (3.10) we have that $\liminf _{n \rightarrow+\infty}\left|\tilde{u}_{n}\right|_{2^{+}}^{2^{+}}>0$. This together with (4.6), (4.7) and the fact that $\tilde{u}_{n} \in \mathcal{N}_{p_{n}^{\prime}}$ gives that $\left(r_{n}\right)$ is bounded. We finish the proof of the claim by applying Lemma 3.1.

Now, using the equality

$$
c_{*}=\inf _{u \in \mathcal{M}_{2^{*}}} I_{2^{*}}(u)=\inf _{u \in \mathcal{N}_{2^{+}}} J_{2^{+}}(u), \quad(\text { see [2]) }
$$

we find

$$
c_{*} \leq J_{2^{+}}\left(r_{n} \tilde{u}_{n}\right) .
$$

Thus, combining the Hölder's inequality with (4.7) and Claim 4.3, we obtain

$$
\begin{aligned}
c_{*} \leq & J_{2^{+}}\left(r_{n} \tilde{u}_{n}\right)=\frac{1}{2^{+}} \int_{\Omega}\left|r_{n} \tilde{u}_{n}\right|^{2^{+}} \mathrm{d} x-\frac{1}{2} \int_{\Omega} K_{2^{+}, \Omega}\left(r_{n} \tilde{u}_{n}\right) r_{n} \tilde{u}_{n} \mathrm{~d} x \\
\leq & \left(\frac{1}{p_{n}^{\prime}}+o_{n}(1)\right)\left[o_{n}(1)+\left(\int_{\Omega}\left|r_{n} \tilde{u}_{n}\right|^{p_{n}^{\prime}} \mathrm{d} x\right)^{\frac{2^{+}}{p_{n}^{\prime}}}\right] \\
& -\frac{1}{2} \int_{\Omega} K_{p_{n}^{\prime}, \Omega}\left(r_{n} \tilde{u}_{n}\right) r_{n} \tilde{u}_{n} \mathrm{~d} x \\
= & o_{n}(1)+\frac{1}{p_{n}^{\prime}}\left(\int_{\Omega}\left|r_{n} \tilde{u}_{n}\right|^{p_{n}^{\prime}} \mathrm{d} x\right)^{\frac{2^{+}}{p_{n}^{\prime}}}-\frac{1}{2} \int_{\Omega} K_{p_{n}^{\prime}, \Omega}\left(r_{n} \tilde{u}_{n}\right) r_{n} \tilde{u}_{n} \mathrm{~d} x \\
= & o_{n}(1)+\frac{1}{p_{n}^{\prime}}\left(\int_{\Omega}\left|r_{n} \tilde{u}_{n}\right|^{p_{n}^{\prime}} \mathrm{d} x\right)^{1+o_{n}(1)}-\frac{1}{2} \int_{\Omega} K_{p_{n}^{\prime}, \Omega}\left(r_{n} \tilde{u}_{n}\right) r_{n} \tilde{u}_{n} \mathrm{~d} x \\
= & o_{n}(1)+\frac{1}{p_{n}^{\prime}} \int_{\Omega}\left|r_{n} \tilde{u}_{n}\right|^{p_{n}^{\prime}} \mathrm{d} x-\frac{1}{2} \int_{\Omega} K_{p_{n}^{\prime}, \Omega}\left(r_{n} \tilde{u}_{n}\right) r_{n} \tilde{u}_{n} \mathrm{~d} x \\
= & o_{n}(1)+J_{p_{n}^{\prime}}\left(r_{n} \tilde{u}_{n}\right) \\
\leq & o_{n}(1)+J_{p_{n}^{\prime}}\left(\tilde{u}_{n}\right)=o_{n}(1)+c_{*} .
\end{aligned}
$$

Consequently, $w_{n}:=r_{n} \tilde{u}_{n} \in \mathcal{N}_{2}$ satisfies $J_{2+}\left(w_{n}\right) \rightarrow c_{*}$. Without loss of generality, using the Ekeland's Variational Principle, we can assume that $w_{n}$ also satisfies $J_{2^{+}}^{\prime}\left(w_{n}\right) \rightarrow 0$ as $n \rightarrow \infty$, that is, $\left(w_{n}\right)$ is a $(P S)$ sequence for $J_{2^{+}}$at the level $c_{*}$. 
By Lemma 3.9, there is a $(P S)$ sequence $\left(v_{n}\right) \subset \mathcal{M}_{2 *}$ for $I_{2 *}$ at the level $c_{*}$. Observe that $\left(v_{n}\right)$ satisfies

$$
\frac{\int_{\Omega}\left|\nabla v_{n}\right|^{2} \mathrm{~d} x}{\left(\int_{\Omega}\left|v_{n}\right|^{2^{*}} \mathrm{~d} x\right)^{\frac{2}{2^{*}}}}=\left(\int_{\Omega}\left|v_{n}\right|^{2^{*}} \mathrm{~d} x\right)^{1-\frac{2}{2^{*}}}=\left(\int_{\Omega}\left|v_{n}\right|^{2^{*}} \mathrm{~d} x\right)^{\frac{2}{N}}
$$

and

$$
c_{*}+o_{n}(1)=I_{2^{*}}\left(v_{n}\right)=\left(\frac{1}{2}-\frac{1}{2^{*}}\right) \int_{\Omega}\left|v_{n}\right|^{2^{*}} \mathrm{~d} x=\frac{1}{N} \int_{\Omega}\left|v_{n}\right|^{2^{*}} \mathrm{~d} x,
$$

that is,

$$
\lim _{n \rightarrow \infty} \int_{\Omega}\left|v_{n}\right|^{2^{*}} \mathrm{~d} x=N c_{*}=S^{N / 2} .
$$

By (4.8) and (4.9),

$$
\lim _{n \rightarrow \infty} \frac{\int_{\Omega}\left|\nabla v_{n}\right|^{2} \mathrm{~d} x}{\left(\int_{\Omega}\left|v_{n}\right|^{2^{*}} \mathrm{~d} x\right)^{\frac{2}{2^{*}}}}=S .
$$

By setting the function $w_{n}=\frac{v_{n}}{\left|v_{n}\right|_{2^{*}}}$, we have that

$$
\left|w_{n}\right|_{2^{*}}=1 \text { and } \lim _{n \rightarrow+\infty} \int_{\Omega}\left|\nabla w_{n}\right|^{2} \mathrm{~d} x=S .
$$

Arguing as in [32, Lemma 5.23], we can apply the Concentration-Compactness Lemma due to Lions [32, Lema 1.40] to find $u \in D^{1,2}\left(\mathbb{R}^{N}\right)$ and a subsequence of $\left(u_{n}\right)$, still denoted by itself, such that

$$
\begin{aligned}
w_{n} \rightarrow u \text { in } & D^{1,2}\left(\mathbb{R}^{N}\right), \\
\left|\nabla w_{n}\right|^{2} \rightarrow \mu & \text { in } \quad \mathcal{M}\left(\mathbb{R}^{N}\right),
\end{aligned}
$$

and

$$
\left|w_{n}\right|^{2^{*}} \rightarrow v \text { in } \mathcal{M}\left(\mathbb{R}^{N}\right)
$$

where $\mu$ and $v$ are positive finite measure with $v$ concentrated at a single point $y \in \bar{\Omega}$. Therefore,

$$
\int_{\Omega} x\left|w_{n}\right|^{2^{*}} \mathrm{~d} x \rightarrow \int_{\Omega} x d v=y \in \bar{\Omega}
$$

or equivalently

$$
\alpha\left(v_{n}\right):=\frac{\int_{\Omega} x\left|v_{n}\right|^{2^{*}} \mathrm{~d} x}{\int_{\Omega}\left|v_{n}\right|^{2^{*}} \mathrm{~d} x} \rightarrow \int_{\Omega} x d \nu=y \in \bar{\Omega},
$$

implying that

$$
\alpha\left(v_{n}\right) \in \Omega^{+}
$$

for $n$ large enough. Thereby,

$$
\beta\left(u_{n}\right)=\alpha\left(v_{n}\right) \in \Omega^{+},
$$

for $n$ large enough, which contradicts (4.2). This completes the proof.

As a by-product of the last proposition, we have that 
Corollary 4.4 For $\epsilon, p^{*}, \delta_{1}>0$ given in Proposition 4.1, for each $p \in\left(p^{*}, 2^{*}\right), \delta \in\left(0, \delta_{1}\right)$, if $u \in \mathcal{S}_{p^{\prime}}$ satisfies $\Psi_{p^{\prime}, \delta}(u) \leq c_{*}+\epsilon$, then $\beta\left(m_{p^{\prime}, \delta}(u)\right) \in \Omega^{+}$.

Proof Indeed, for fixed $p \in\left(p^{*}, 2^{*}\right), \delta \in\left(0, \delta_{1}\right)$, if $u \in \mathcal{S}_{p^{\prime}}$ is such that $\Psi_{p^{\prime}, \delta}(u) \leq c_{*}+\epsilon$, then $m_{p^{\prime}, \delta}(u) \in \mathcal{N}_{p^{\prime}, \delta}$ with $J_{p^{\prime}, \delta}\left(m_{p^{\prime}, \delta}(u)\right) \leq c_{*}+\epsilon$. By Theorem 4.1, $\beta\left(m_{p^{\prime}, \delta}(u)\right) \in \Omega^{+}$.

The next result establishes a crucial relation between cat $\left(\mathcal{S}_{p^{\prime}}^{c_{*}+\epsilon}\right)$ and cat $(\Omega)$, where

$$
\mathcal{S}_{p^{\prime}}^{c_{*}+\epsilon}=\left\{u \in S_{p^{\prime}}: \Psi_{p^{\prime}, \delta}(u) \leq c_{*}+\epsilon\right\}
$$

Proposition 4.5 For $\epsilon, p^{*}, \delta_{1}>0$ given in Proposition 4.1, $p \in\left(p^{*}, 2^{*}\right)$ and $\delta \in\left(0, \delta_{1}\right)$, we have

$$
\operatorname{cat}\left(\mathcal{S}_{p^{\prime}}^{c_{*}^{*}+\epsilon}\right) \geq \operatorname{cat}(\Omega)
$$

Proof By Proposition 3.5, we can fix $r>0$ such that $c_{p^{\prime}, B_{r}(0)}<c_{*}+\epsilon$. Let $k=\operatorname{cat}\left(\mathcal{S}_{p^{\prime}}^{c_{*}+\epsilon}\right)$. Then, there are $k$ closed contractible sets $A_{j} \subseteq \mathcal{S}_{p^{\prime}}^{c_{*}+\epsilon}, j=1, \ldots, k$ such that $\cup_{j=1}^{k} A_{j}=$ $\mathcal{S}_{p^{\prime}}^{c_{*}+\epsilon}$. This means that there are $k$ continuous applications $h_{j}:[0,1] \times A_{j} \rightarrow \mathcal{S}_{p^{\prime}}^{c_{*}+\epsilon}$ such that

$$
h_{j}(0, u)=u, \quad h_{j}(1, u)=h_{j}(1, v), \forall u, v \in A_{j}, j=1, \ldots, k .
$$

Setting $B_{j}:=\left(m_{p^{\prime}, \delta}^{-1} \circ \Phi_{p^{\prime}, \delta}\right)^{-1}\left(A_{j}\right), j=1, \ldots, k$, we derive that $B_{j}$ are closed and $B_{j} \subset \Omega^{-}$. Moreover, as

$$
\Psi_{p^{\prime}, \delta}\left(\left(m_{p^{\prime}, \delta}^{-1} \circ \Phi_{p^{\prime}, \delta}\right)(y)\right)=J_{p^{\prime}, \delta}\left(\Phi_{p^{\prime}, \delta}(y)\right)=c_{p^{\prime}, B_{r}(0)}<c_{*}+\epsilon, \forall y \in \Omega^{-},
$$

or equivalently

$$
\Psi_{p^{\prime}, \delta}\left(\left(m_{p^{\prime}, \delta}^{-1} \circ \Phi_{p^{\prime}, \delta}\right)\left(\Omega^{-}\right)\right) \subset \mathcal{S}_{p^{\prime}}^{c_{*}+\epsilon}
$$

we also have that

$$
\bigcup_{j=1}^{k} B_{j}=\Omega^{-} \text {. }
$$

Consider the applications $l_{j}:[0,1] \times B_{j} \rightarrow \Omega^{+}$given by

$$
l_{j}(t, x):=\beta \circ m_{p^{\prime}, \delta} \circ h_{j}\left(t, m_{p^{\prime}, \delta}^{-1} \circ \Phi_{p^{\prime}, \delta}(x)\right) .
$$

Then $l_{j}$ is continuous and, for $x, y \in B_{j} \subset \Omega^{+}$, using (4.10) and (4.1),

$$
\begin{aligned}
l_{j}(0, x) & =\beta \circ m_{p^{\prime}, \delta} \circ h_{j}\left(0, m_{p^{\prime}, \delta}^{-1} \circ \Phi_{p^{\prime}, \delta}(x)\right) \\
& =\beta \circ m_{p^{\prime}, \delta} \circ m_{p^{\prime}, \delta}^{-1} \circ \Phi_{p^{\prime}, \delta}(x) \\
& =\beta \circ \Phi_{p^{\prime}, \delta}(x)=x,
\end{aligned}
$$

and

$$
\begin{aligned}
l_{j}(1, x) & =\beta \circ m_{p^{\prime}, \delta} \circ h_{j}\left(1, m_{p^{\prime}, \delta}^{-1} \circ \Phi_{p^{\prime}, \delta}(x)\right) \\
& =\beta \circ m_{p^{\prime}, \delta} \circ h_{j}\left(1, m_{p^{\prime}, \delta}^{-1} \circ \Phi_{p^{\prime}, \delta}(y)\right) \\
& =l_{j}(1, y) .
\end{aligned}
$$


Thus, $B_{j}$ are contractible and by (4.11),

$$
\operatorname{cat}(\Omega)=\operatorname{cat}_{\Omega^{+}}\left(\Omega^{-}\right) \leq k=\operatorname{cat}\left(\mathcal{S}_{p^{\prime}}^{c_{*}+\epsilon}\right),
$$

as desired.

Proof of Theorem 1.1 Let $p \in\left(p^{*}, 2^{*}\right)$ and $0<\delta<\delta^{*}:=\min \left\{\delta_{0}, \delta_{1}\right\}$. Then, by Lemma 3.7-(iv), $c_{*}+\epsilon>c_{*}=\inf _{\mathcal{S}_{p^{\prime}}} \Psi_{p^{\prime}, \delta}$. This and Corollary 3.8 allow us to apply the LusternikSchnirelmann category to $\Psi_{p^{\prime}, \delta}$, which guarantee us that $\Psi_{p^{\prime}, \delta}$ has at least $\operatorname{cat}\left(\mathcal{S}_{p^{\prime}}^{c_{*}+\epsilon}\right)$ nontrivial critical points on $\mathcal{S}_{p^{\prime}}^{c_{*}+\epsilon}$. Applying Lemma 3.7-(iv) and Proposition 4.5, we conclude that $J_{p^{\prime}, \delta}$ has at least cat $(\Omega)$ nontrivial critical points. Thus, by Theorem $2.1,\left(P_{p, \delta}\right)$ has at least cat $(\Omega)$ nontrivial solutions. Moreover, since $f$ is odd, Corollary 3.3 together with maximum principle yields these solutions can be chosen positive.

Acknowledgements The authors would like to thank the referee for his/her important comments.

\section{References}

1. Ambrosetti, A., Rabinowitz, P.H.: Dual variational methods in critical point theory and applications. J. Funct. Anal. 44, 349-381 (1973)

2. Ambrosetti, A., Turner, R.E.L.: Some discontinuous variational problems. Differ. Int. Equ. 1, 341-349 (1988)

3. Ambrosetti, A., Struwe, M.: A note on the problem $-\Delta u=\lambda u+u|u|^{2^{*}-2}$. Manuscr. Math. 54, 373-379 (1986)

4. Ambrosetti, A., Badiale, M.: The dual variational principle and elliptic problems with discontinuous nonlinearities. J. Funt. Anal. 140, 363-373 (1989)

5. Ambrosetti, A., Calahorrano, M., Dobarro, F.: Global branching for discontinuous problems. Comment. Math. Univ. Carol. 31, 213-222 (1990)

6. Alves, C.O., Bertone, A.M., Gonçalves, J.V.: A variational approach to discontinuous problems with critical Sobolev exponents. J. Math. Anal. Appl. 265, 103-127 (2002)

7. Alves, C.O., Bertone, A.M.: A discontinuous problem involving the $p$-Laplacian operator and critical exponent in $\mathbb{R}^{N}$. Electron. J. Differ. Equ. 2003, 1-10 (2003)

8. Alves, C.O., Gonçalves, J.V., Santos, J.A.: Strongly nonlinear multivalued elliptic equations on a bounded domain. J. Glob. Optim. 58, 565-593 (2014)

9. Alves, C.O., Ding, Y.H.: Multiplicity of positive solutions to a p-Laplacian equation involving critical nonlinearity. J. Math. Anal. Appl. 279, 508-521 (2003)

10. Alves, C.O., Carrião, P.C., Miyagaki, O.H.: On the existence of positive solutions of a perturbed Hamiltonian system in $\mathbb{R}^{N}$. J. Math. Anal. Appl. 276, 673-690 (2002)

11. Alves, C.O., Nóbrega, A.B.: Nodal ground state solution to a biharmonic equation via dual method. J. Differ. Equ. 260, 5174-5201 (2016)

12. Badiale, M., Tarantello, G.: Existence and multiplicity results for elliptic problems with critical growth and discontinuous nonlinearities. Nonlinear Anal. 29, 639-677 (1997)

13. Bahri, A., Coron, J.M.: On a nonlinear elliptic equation involving the critical Sobolev exponent: the effect of the topology of the domain. Commun. Pure Appl. Math. 41, 253-294 (1988)

14. Benci, V., Cerami, G.: The effect of the domain topology on the number of positive solutions of nonlinear elliptic problems. Arch. Ration. Mech. Anal. 114, 79-93 (1991)

15. Benci, V., Cerami, G.: Multiple positive solutions of some elliptic problems via the Morse theory and the domain topology. Calc. Var. Partial Differ. Equ. 2, 29-48 (1994)

16. Carl, S., Le, V.K., Motreanu, D.: Nonsmooth Variational Problems and Their Inequalities. Comparison Principles and Applications. Springer Monographs in Mathematics. Springer, New York (2007)

17. Clarke, F.H.: Optimization and Nonsmooth Analysis. Wiley, New York (1983)

18. Chang, K.C.: Variational methods for nondifferentiable functionals and their applications to partial differential equations. J. Math. Anal. Appl. 80, 102-129 (1981)

19. Chang, K.C.: On the multiple solutions of the elliptic differential equations with discontinuous nonlinear terms. Sci. Sin. 21, 139-158 (1978) 
20. Chang, K.C.: The obstacle problem and partial differential equations with discontinuous nonlinearities. Commun. Pure Appl. Math. 33, 117-146 (1980)

21. Carl, S., Dietrich, H.: The weak upper and lower solution method for elliptic equations with generalized subdifferentiable perturbations. Appl. Anal. 56, 263-278 (1995)

22. Carl, S., Heikkila, S.: Elliptic equations with discontinuous nonlinearities in $\mathbb{R}^{N}$. Nonlinear Anal. 31, 217-227 (1998)

23. Carl, S., Heikkila, S.: Elliptic equations with discontinuous nonlinearities in $\mathbb{R}^{N}$. Nonlinear Anal. 30, 1743-1751 (1997)

24. Cerami, G.: Metodi variazionalli nello studio di problemi al contorno con parte nonlineare discontinua. Rend. Circ. Mat. Palermo 32, 336-357 (1983)

25. Hu, S., Kourogenis, N., Papageorgiou, N.S.: Nonlinear elliptic eigenvalue problems with discontinuities. J. Math. Anal. Appl. 233, 406-424 (1999)

26. Kavian, O.: Introduction á la théorie des points critiques et applications aux problémes elliptíques. Springer, Heidelberg (1993)

27. Motreanu, D., Varga, C.: Some critical point results for locally Lipschitz functionals. Commun. Appl. Nonlinear Anal. 4, 17-33 (1997)

28. Radulescu, V.: Mountain pass theorems for non-differentiable functions and applications. Proc. Jpn. Acad. (Ser. A) 69, 193-198 (1993)

29. Rey, O.: A multiplicity result for a variational problem with lack of compactness. Nonlinear Anal. TMA 13, 1241-1249 (1989)

30. Szulkin, A., Weth, T.: The method of Nehari manifold. In: Gao, D.Y., Montreanu, D. (eds.) Handbook of Nonconvex Analysis and Applications, pp. 597-632. International Press, Boston (2010)

31. Struwe, M.: A global compactness result for elliptic boundary value problem involving limiting nonlinearities. Math. Z. 187, 511-517 (1984)

32. Willem, W.: Minimax Theorems. Birkhauser, Basel (1986) 